\title{
Nanohybrid platform based on carbon material for glucose detection
}

\author{
$\underline{\text { Maria Kuznowicz }}^{1}$, Artur Jędrzak ${ }^{2}$, Teofil Jesionowski ${ }^{3}$ \\ 1. Institute of Chemical Technology and Engineering, Faculty of Chemical Technology, Poznan University of \\ Technology, POLAND, Poznań, Berdychowo 4, PL-60965, E-mail: maria.m.kuznowicz@ doctorate.put.poznan.pl \\ 2. Institute of Chemical Technology and Engineering, Faculty of Chemical Technology, Poznan University of \\ Technology, POLAND, Poznań, Berdychowo 4, PL-60965, E-mail: artur.jedrzak@put.poznan.pl \\ 3. Institute of Chemical Technology and Engineering, Faculty of Chemical Technology, Poznan University of \\ Technology, POLAND, Poznań, Berdychowo 4, PL-60965, E-mail: teofil.jesionowski@ put.poznan.pl
}

The main goal of the activities was to create a hybrid nanoplatform decorated with copper oxide. The produced matrix was used for the non-enzymatic detection of glucose in model solutions and blood serum.

sensor, non-enzymatic sensor, nanomaterials, hybrid material, glucose detection, amperometric sensor

\section{Introduction (use Times New Roman, 12, bold, center)}

Sensors are becoming increasingly common in food and biomedical industries, as well as in clinical diagnostics, biological, and environmental applications. Glucose sensors, in particular, are critical for diabetes diagnosis and tracking food quality. Sensors are instruments made up of active sensing materials coupled with a transducer of a signal. These devices transmit the signal from a reaction shift or selective compound and generate a signal (such as electrical, thermal, or optical output signals) that is transformed for further processing into digital signals. Because of their outstanding electro-catalytic activity, selectivity, and sensitivity, amperometric glucose biosensors using glucose oxidase have been widely investigated for the detection of glucose levels in blood and food products [1].

Enzyme-based biosensors, on the other hand, have drawbacks such as unpredictable responses caused by changes in $\mathrm{pH}$, temperature, humidity, and the presence of interfering chemicals. Additional drawbacks include enzyme leaching, direct electron transfer between the active sites of enzymes (flavin adenine dinucleotide or FAD), and electrode replacement difficulties due to the redox centre of FAD being deeply embedded in enzyme molecules. An alternative strategy based on direct electro-oxidation with non-enzymatic glucose sensors has been proposed to address these challenges. The main aim was to find a suitable catalyst with direct electrochemical activity and to resolve the limitations of currently available enzymatic glucose sensors [2].

The majority of non-enzymatic glucose sensors investigated are focused on noble metal substrates such as platinum, gold, palladium, alloys of $\mathrm{Pt}, \mathrm{Pd}, \mathrm{Pb}$, and $\mathrm{Rh}$, metals such as copper, nickel, zinc, manganese, metal compounds, metal oxide composites, polymer-modified composites, carbon nanotubes, carbon nanofibers, graphene, and their composites, doped diamond-like materials, and nanodiamonds, as well as carbon nanotubes. Due to their high electrochemical activity, non-toxicity, low cost, and potential integration with other materials, metals/metal oxides, especially copper and its oxides, are promising for the preparation of nonenzymatic glucose sensors. Copper and copper oxide-based nanomaterials have been studied for their glucose sensing properties as well as for improving the catalytic activity of other nanomaterials used as support matrices [3]. 
In this work, a hybrid material was produced based on multi-wall carbon nanotubes and a polymer coating. The material was then decorated with copper(II) oxide using electrodeposition. The schematic steps of the process are presented in the Fig. 1.

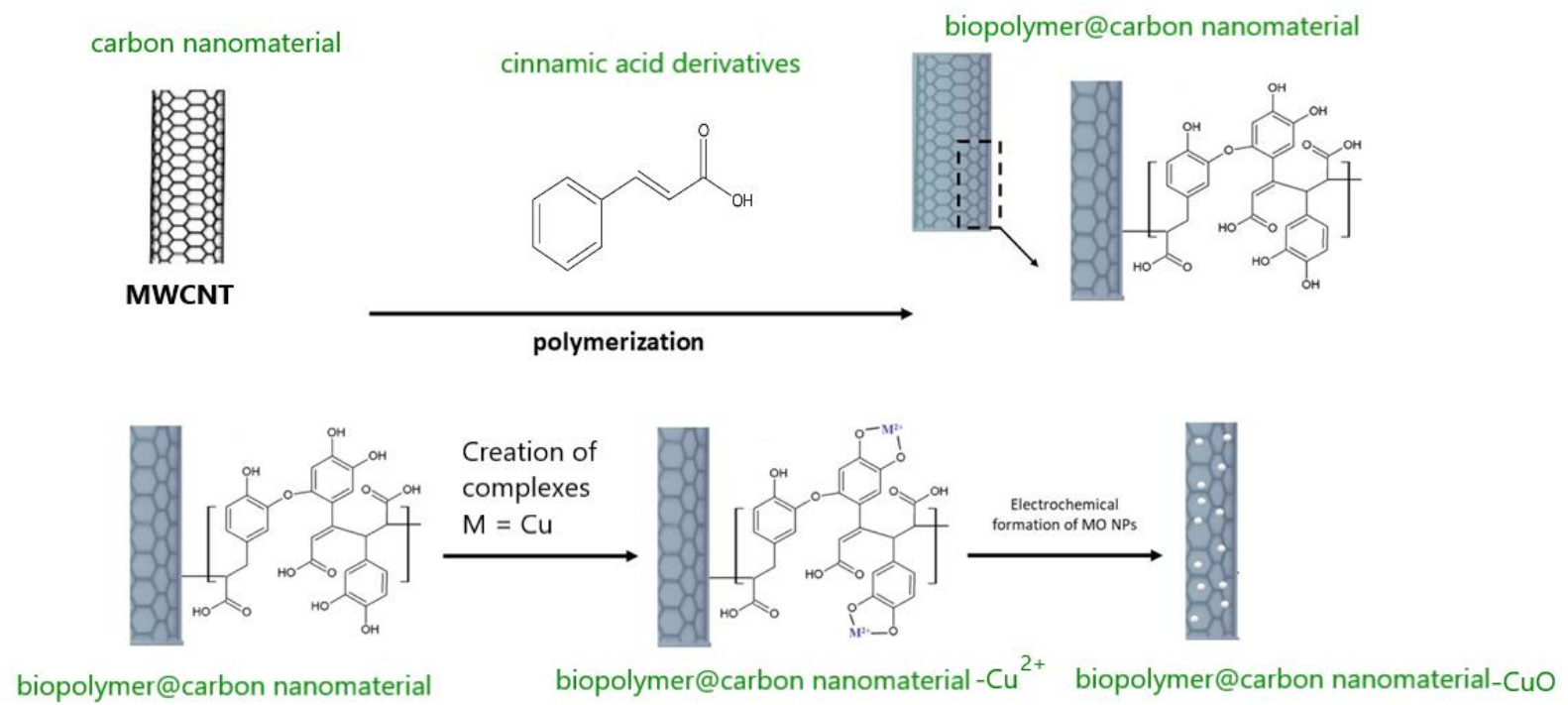

Fig.1. Schematic representation of the activities performed as part of the experiment

Besides, good accuracy and high precision have been demonstrated for quantifying glucose concentrations in human serum samples. Observed good features of the proposed sensor have great potential in creating sensitive and selective non-enzymatic glucose sensors.

\section{Conclusion}

In this work, the novel nanohybrid platform has been proposed. Copper(II) oxide was produced by the electrodeposition process on the matrix surface. This made it possible to use the matrix as a non-enzymatic glucose sensor. The proposed sensor was characterized by good analytical properties and was successfully used to detect glucose in blood serum.

\section{Acknowledgments}

This work was financed and prepared as part of a research project supported by the National Science Center Poland, no. 2017/27/B/ST8/01506.

\section{References}

[1] Sridara T., Upan J., Saianand G., Tuantranont A., Kuruwan C., Jakmunee J. (2020) Nonenzymatic amperometric glucose sensor based on carbon nanodots and copper oxide nanocomposites electrode, 20,808. doi: 10.3390/s20030808.

[2] Batool R., Rhouati A., Nawaz M.H., Hayat A., Marty J.L (2019) A review of the constriction of nano-hybrids for electrochemical biosensing of glucose, Biosensors 9, 46, doi:10.3390/bios9010046

[3] Carbone M., Tagliatesta P. (2020) NiO grained-flowers and nanoparticles for ethanol sensing, Materials, 13,1880. doi:10.3390/ma13081880. 